\title{
An Efficient Signed-Power-of-Two Term Allocation for Filter Coefficients in Digital Communication System
}

\author{
Koichi ICHIGE $^{\dagger a)}$, Member, Hideaki MUNEMASA ${ }^{\dagger}$, Student Member, and Hiroyuki ARAI ${ }^{\dagger}$, Member $^{\circ}$
}

\begin{abstract}
SUMMARY This letter presents an efficient Signed-Power-of-Two (SPT) term allocation for filter coefficients in order to improve the BER characteristics of digital communication systems. The performance of the present allocation is evaluated by BER characteristics through digital modulation simulations and FPGA-based digital implementation.

key words: digital modulation, BER characteristics, cosine rolloff filter, SPT term
\end{abstract}

\section{Introduction}

In multichannel digital communication system, interference from adjacent frequency channels should be reduced in order to achieve good Bit Error Rate (BER) characteristics for the efficient use of the limited frequency bands. Nyquist digital filters whose impulse response cross zero at every symbol interval are employed for frequency band restriction in the transmitter and interference reduction in the receiver as depicted in Fig. 1. Cosine Rolloff Filter (CRF) or root-CRF are representative of the Nyquist filters used in baseband or IF signal processing for various kinds of communication applications. Such filters are very common devices and generally are not paid so much attention, but they are used in almost all the digital communication system including Software Defined Radio (SDR); most of the SDR transmitters and receivers employ analog and/or digital lowpass filters in order to restrict the frequency bandwidth of signals to a certain short range.

The filter characteristics tend to become worse if we implement the filter on a fixed-point digital device such as Digital Signal Processor (DSP) or Field Programmable Gate Array (FPGA) with shorter bit length quantization. An effective quantization way for filter coefficients would be the use of Signed-Power-of-Two (SPT) term [1], [2]. Representing each filter coefficient as a sum of SPT $\left( \pm 2^{n}\right)$ term, the designed filter can be implemented with very small circuit capacity while preserving good frequency characteristics. The literatures [1], [2] mention the way to allocate SPT term to obtain overall good amplitude characteristics, but this allocation is not always the best for communication systems which are evaluated through BER characteristics.

This letter focuses the way of SPT term allocation to

\footnotetext{
Manuscript received March 29, 2006.

Manuscript revised June 30, 2006.

${ }^{\dagger}$ The authors are with the Department of Electrical and Computer Engineering, Yokohama National University, Yokohama-shi, 240-8501 Japan.

a)E-mail: koichi@ynu.ac.jp

DOI: 10.1093/ietcom/e89-b.12.3266
}

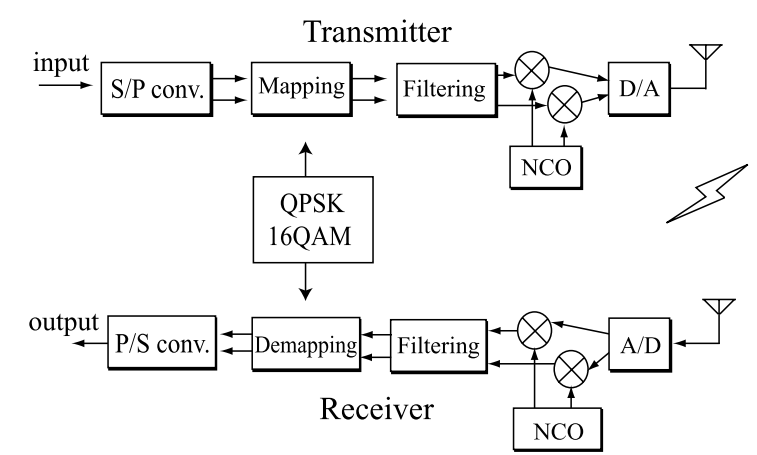

Fig. 1 Basic configuration of transmitter and receiver in wireless digital communication system.

improve BER characteristics in communication system. We develop an efficient allocation which allocates more SPT term to the edge of impulse response, and less to the center by introducing weight parameters. We design some rootCRFs and evaluate BER characteristics when those filters are employed in digital modulation scheme.

\section{SPT Terms Allocation}

\subsection{Conventional Allocation}

Consider an Finite Impulse Response (FIR) filter of order $N$, and let $\left\{h_{n}\right\}_{n=0}^{N-1}$ be the impulse response of the filter. For the given number of total SPT term $R>0$, the conventional SPT Terms allocation [2] can be summarized as follows.

1. Let $u_{n}$ be the number of SPT term corresponding to the impulse response $h_{n}$. Initialize $\left\{u_{n}\right\}_{n=0}^{N-1}$, i.e., $u_{n}=0$ for $n=0,1, \ldots, N-1$.

2. Calculate $c_{n}=0.36 \log _{2}\left|h_{n}\right|$ for $n=0,1, \ldots, N-1$.

3. Find $i \in\{0,1, \ldots, N-1\}$ which minimize $c_{n}$, i.e., $i=$ $\operatorname{argmin} c_{n}$. Then operate the followings.

$n$

$$
\begin{aligned}
& u_{i}+1 \rightarrow u_{i}, \\
& c_{i}-1 \rightarrow c_{i}, \\
& R-1 \rightarrow R .
\end{aligned}
$$

4. End if $R=0$. Otherwise go back to the procedure 3 .

This procedure tends to allocate SPT term mainly in the center of the impulse response as shown in Fig. 2(b). It keeps good characteristics in the sense of filter performance in frequency domain, but this allocation is not always the best 


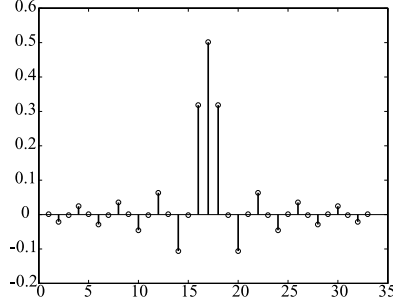

(a) original

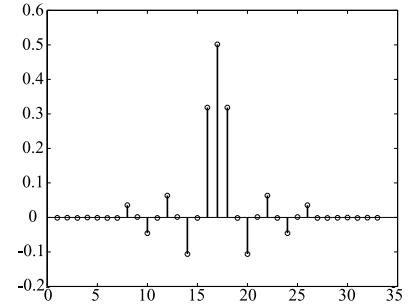

(b) conventional allocation

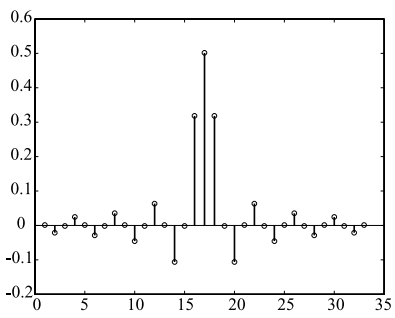

(c) proposed allocation

Fig. 2 Example impulse responses of FIR root-CRF when the symbol rate is $2, N=35$ and $R=22$.

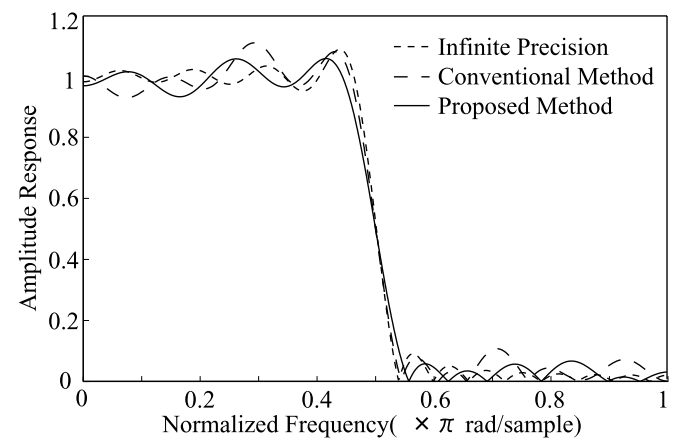

Fig. 3 Example amplitude characteristics of FIR filters in Fig. 2.

way to obtain good BER characteristics in communication system when applied to the coefficients of CRFs or rootCRFs.

\subsection{Proposed Allocation}

In order to allocate more SPT term to the edge of impulse response, we put weight parameter in calculating $c_{n}$ in the above procedure. Since it becomes very complicated to determine the weight so that the BER characteristics is optimized, we tested hundreds of different simple forms of weights and found that the following weight representation is suitable for CRF and root-CRF coefficients used in digital modulation system.

$$
w_{n}=\left(\left\lceil\frac{N}{2}\right\rceil-|n|\right)^{3}+\left\lceil\frac{N}{2}\right\rceil,
$$

where $\lceil a\rceil$ denotes a minimum integer not smaller than $a$. Using the above weight, we modify the SPT term allocation procedure as follows. This procedure could allocate SPT term to the edge of impulse response as seen in Fig. 2(c).

1. Same to the procedure 1. in Subsection 2.1.
Table 1 Specifications of simulation

\begin{tabular}{|c||c|}
\hline Modulation type & $\begin{array}{c}\text { Desired: QPSK / 16QAM } \\
\text { Interference: 8PSK }\end{array}$ \\
\hline Frequency Range & $\begin{array}{c}\text { Desired: [0 MHz, 10 MHz] } \\
\text { Interference: }[10 \mathrm{MHz}, 20 \mathrm{MHz}]\end{array}$ \\
\hline CIR & $0[\mathrm{~dB}]$ \\
\hline Sampling frequency & $40 \mathrm{MHz}$ \\
\hline Filter type & Root CRF, lowpass \\
\hline Filter order $N$ & 35 \\
\hline Cutoff frequency & $10[\mathrm{MHz}]$ \\
\hline Rolloff Rate & 0.5 \\
\hline \# of SPT Terms & 30 \\
\hline
\end{tabular}

2. Calculate $c_{n}=0.36 \log _{2}\left|w_{n} h_{n}\right|$ for $n=0,1, \ldots, N-1$.

3. Same to the procedure 3. in Subsection 2.1.

4. Same to the procedure 4. in Subsection 2.1.

Figure 3 shows example amplitude characteristics of the designed FIR root CRFs in Fig. 2. The amplitude characteristics are almost similar to each other at a glance but that by Ref. [2] has some relatively large ripples in pass- and stop-bands in comparison with the result by the proposed method. It may be negligible from filter characteristics but still affects to digital communication quality.

\section{Simulation}

The proposed SPT term allocation is evaluated through computer simulation whose specifications are summarized in Table 1. We assume the case that the frequency band for desired signal and that for interference are next to each other, and demonstrate to suppress the interference.

Figure 4 shows the BER characteristics for (a) QPSK signals and (b) 16QAM signals. Note that the case of 2-bit quantization has almost the same computational complexity when $N=35$ and $R=30$. We see that the BER characteristics by the proposed SPT term allocation is more effective than the other quantization methods, and realize almost the same performance with infinite precision. Especially when BER stays around $10^{-4}$ to $10^{-5}$, means the significant range for high quality communication, the proposed allocation greatly improves BER characteristics. Note that we tested various situations (modulation type, frequency, filter order, number of SPT term) and confirmed the similar improvements, however space does not permit to show them all.

\section{Implementation}

A QPSK/16QAM demodulator using root-CRF developed in Sections 2 and 3 is implemented on FPGA-based digital device [3]. Table 2 shows the specifications of the digital processor; those for signals and filters are same with Table 1. Also Fig. 5 describes an overview of the processing flow on digital processor.

Table 3 compares the circuit scale when all the digital demodulation and related processes are implemented on FPGA. The SPTterm-based implementation requires small 


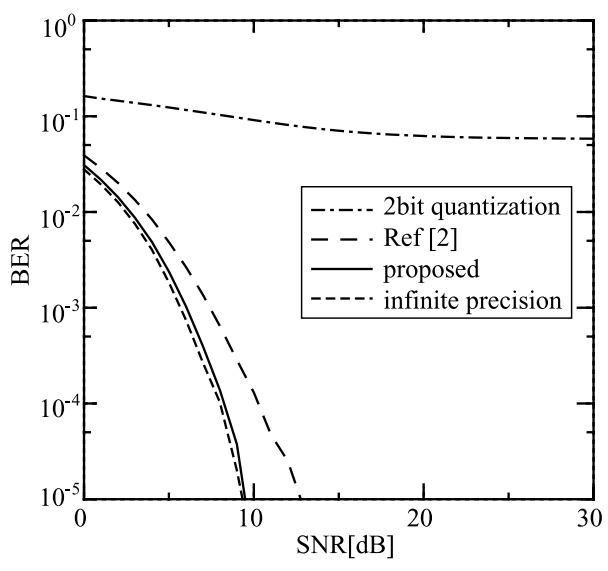

(a) For QPSK signals.

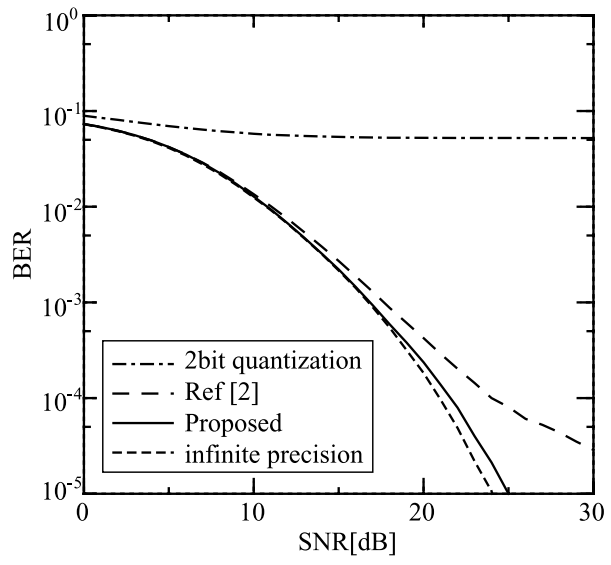

(b) for 16QAM signals

Fig. 4 BER characteristics for QPSK and 16QAM signals.

Table 2 Specification of FPGA-based QPSK/16QAM demodulator.

\begin{tabular}{|c||c|}
\hline A/D converter & $40 \mathrm{MHz} / 12$ bit sampling \\
\hline CPU & Hitachi SH4, 200 MHz \\
\hline FPGA & Altera Stratix EP1S25 $(600,000$ gates $)$ \\
\hline Interface & TCP/IP Ethrenet port \\
\hline
\end{tabular}

number of logic elements and no any multiplier since shift register can be used instead, while the direct implementation requires multiplication (DSP block on FPGA) for the convolution of impulse response and large number of logic elements. We see that the proposed allocation en-

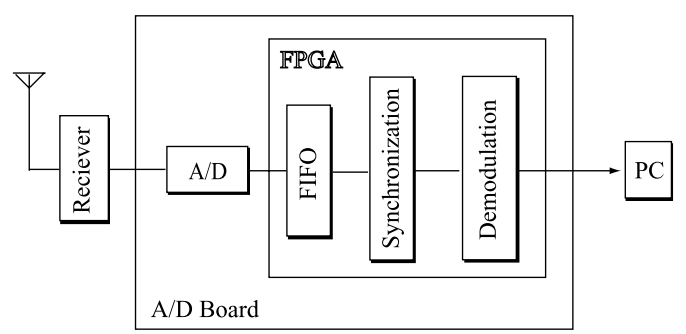

Fig.5 Processing flow of FPGA-based QPSK/16QAM demodulator.

Table 3 Comparison of circuit scale.

\begin{tabular}{|c|c|c|}
\hline & $\begin{array}{c}\text { direct } \\
\text { implementation }\end{array}$ & $\begin{array}{c}\text { SPTterm-based } \\
\text { implementation }\end{array}$ \\
\hline Logic elements & 893 & 293 \\
\hline Clock frequency & $67.9 \mathrm{MHz}$ & $74.4 \mathrm{MHz}$ \\
\hline \# of DSP blocks & 33 & 0 \\
\hline
\end{tabular}

ables small circuit capacity, multiplierless implementation and high clock frequency. Also note that the BER characteristics obtained by the experiments completely overlaps the simulation results in Fig. 4.

\section{Concluding Remarks}

This letter presented an effective SPT term allocation for filter coefficients in order to improve BER characteristics in digital communication system. The present allocation was evaluated through computer simulation and FPGA-based digital implementation, and found that the proposed SPT term allocation is effective for digital modulation system.

\section{References}

[1] J.J. Shyu and Y.C. Lim, "A new approach to the design of discrete coefficient FIR digital filters," IEEE Trans. Signal Process., vol.43, no.1, pp.310-314, Jan. 1995.

[2] Y.C. Lim, R. Yang, D. Li, and J. Song, "Signed power-of-two terms allocation scheme for the design of digital filters," IEEE Trans. Circuits Syst. II, vol.46, no.5, pp. 577-584, May 1999.

[3] M. Kim, A. Kiyono, K. Ichige, and H. Arai, "Experimental study of jitter effect on digital downconversion receiver with undersampling scheme," IEICE Trans. Inf. \& Syst., vol.E88-D, no.7, pp.14301436, July 2005. 